\title{
Outbreak of Salmonella Oslo Infections Linked to Persian Cucumbers - United States, 2016
}

\begin{abstract}
Lyndsay Bottichio, $\mathrm{MPH}^{1}$; Carlota Medus, $\mathrm{PhD}^{2}$; Alida Sorenson, $\mathrm{MPH}^{3}$; Danielle Donovan, MS ${ }^{4}$; Reeti Sharma, MPH${ }^{5}$; Natasha Dowell, MPH${ }^{1}$; Ian Williams, $\mathrm{PhD}^{1}$; Allison Wellman, $\mathrm{MPH}^{6}$; Alikeh Jackson, $\mathrm{MPH}^{6}$; Beth Tolar, $\mathrm{MPH}^{1}$; Taylor Griswold, MS ${ }^{1}$; Colin Basler, DVM ${ }^{1}$
\end{abstract}

In April 2016, PulseNet, the national molecular subtyping network for foodborne disease surveillance, detected a multistate cluster of Salmonella enterica serotype Oslo infections with an indistinguishable pulsed-field gel electrophoresis (PFGE) pattern (XbaI PFGE pattern OSLX01.0090).* This PFGE pattern was new in the database; no previous infections or outbreaks have been identified. CDC, state and local health and agriculture departments and laboratories, and the Food and Drug Administration (FDA) conducted epidemiologic, traceback, and laboratory investigations to identify the source of this outbreak. A total of 14 patients in eight states were identified, with illness onsets occurring during March 21-April 9, 2016. Whole genome sequencing, a highly discriminating subtyping method, was used to further characterize PFGE pattern OSLX01.0090 isolates. Epidemiologic evidence indicates Persian cucumbers as the source of Salmonella Oslo infections in this outbreak. This is the fourth identified multistate outbreak of salmonellosis associated with cucumbers since 2013 . Further research is needed to understand the mechanism and factors that contribute to contamination of cucumbers during growth, harvesting, and processing to prevent future outbreaks.

\section{Epidemiologic Investigation}

State and local public health officials in Minnesota and Michigan initiated an investigation when four persons with Salmonella Oslo infections were identified. A case was defined as infection with Salmonella Oslo with PFGE pattern OSLX01.0090 (the outbreak strain) in a person with illness onset occurring during March 21-April 9, 2016. Most people infected with Salmonella develop diarrhea, fever, and abdominal cramps 12-72 hours after infection. Initial interviews of ill persons found that shopping at a national chain grocer (chain A) and purchasing produce was commonly reported. A structured, focused supplemental questionnaire was developed to collect detailed information on exposure to grocery stores and produce, including cucumbers and leafy greens, in the 7 days before illness onset. Exposure frequencies were compared with the 2006-2007 FoodNet Population Survey, in which healthy persons reported foods consumed in the week before interview. ${ }^{\dagger}$ Information also was collected on illness subclusters, defined as two or more

\footnotetext{
*http://www.cdc.gov/pulsenet.

$\dagger$ http://www.cdc.gov/foodnet/studies/population-surveys.html.
}

unrelated ill persons who reported eating at the same restaurant, attending the same event, or shopping at the same grocery store in the week before becoming ill.

A total of 14 cases were reported from eight states $\$$ (Figure 1). Illness onset dates ranged from March 21April 9, 2016 (Figure 2). Median age of patients was 36 years (range $=3-68$ years); nine were female. Three patients were hospitalized; no deaths were reported. Thirteen patients were interviewed using the supplemental questionnaire about exposures in the week before illness onset, of whom 12 reported eating cucumbers in the week before becoming ill. Patients were significantly more likely to report consuming cucumbers compared with the 2006-2007 FoodNet Population Survey, in which $46.9 \%$ of respondents reported consuming cucumbers in the week before interview $(\mathrm{p}<0.001)$. Among the 12 patients who consumed cucumbers, 11 specifically reported Persian or "mini" cucumbers, which are small, seedless cucumbers with smooth skin. Eight of 13 respondents reported purchasing their cucumbers from chain A. The proportion of ill persons who reported eating fruit, leafy greens, or any other item on the supplemental questionnaire was not significantly higher than expected when compared with the FoodNet Population Survey.

\section{Traceback Investigation}

FDA, the Canadian Food Inspection Agency, and officials in Minnesota, Massachusetts, and Michigan collaborated to conduct an informational traceback investigation from retail establishments in these states to identify the source of the cucumbers. Informational traceback can support the epidemiologic investigation by quickly assessing the plausibility of one or more vehicles as the source of the outbreak. Informational traceback typically can be completed much more quickly than regulatory traceback, which requires the collection of specific types of records, such as receipts and invoices, at each step of the distribution chain. Factors used to identify the best Salmonella Oslo cases for traceback included confirmed cucumber purchase information, geographic diversity, and diversity of establishments (grocery stores and cucumber suppliers). In addition, an informational traceback was conducted from points of service for patients with reported cucumber

\$Illinois, Kentucky, Massachusetts, Michigan, Minnesota, Nebraska, Ohio, and Wisconsin. 
FIGURE 1. Number of persons $(n=14)$ infected with the outbreak strain of Salmonella Oslo, March 2-April 9, 2016

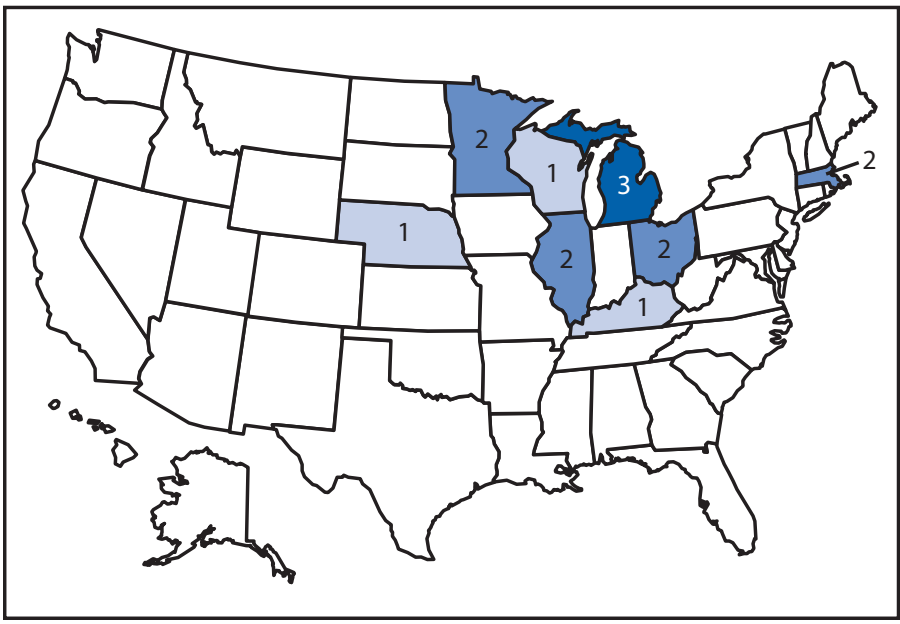

exposures, but without supporting shopper records or receipts. The investigation identified two Canadian Persian cucumber suppliers during the timeframe of interest, but a single grower was not identified. Growers who could have supplied these cucumbers were located in Canada, Mexico, and the Dominican Republic. These Canadian-grown cucumbers would have also been distributed in Canada and no reported clinical cases matching the U.S. outbreak pattern were identified. Only one illness subcluster was identified (in Minnesota), involving two unrelated persons who shopped at the same grocery store and purchased the same brand of Persian cucumbers on the same day. During the informational traceback, it was found that cases in Minnesota and Massachusetts had purchased the same brand of cucumbers from both chain A and a separate chain grocer (chain B). Additional cases from other states also reported purchasing Persian cucumbers from chain A, but could not remember the brand. Further traceback revealed that the cucumbers purchased at both of these chain grocers were sourced from a common produce supplier. These findings indicate that although the majority of patients purchased cucumbers from chain A, chain A was unlikely to be the only venue at which contaminated cucumbers were sold. Chain A voluntarily removed all Persian cucumber products from their shelves while the investigation and traceback efforts were ongoing.

\section{Laboratory Investigation}

Cucumber samples were collected from the point of sale, from patients' homes, and from one of the Canadian suppliers, approximately 1 month after the patients' purchase date, but no cucumbers yielded Salmonella.

Whole genome sequencing was performed on four clinical isolates by state health departments and CDC to further

\section{Summary}

What is already known about this topic?

Salmonella is the most common bacterial cause of foodborne disease in the United States and results in the highest number of hospitalizations and deaths among foodborne pathogens. The Oslo serotype is rare, with about 25 cases reported each year nationally. According to the National Outbreak Reporting System, Salmonella outbreaks associated with cucumbers have been increasing in number each year since 2010.

What is added by this report?

In April 2016, a multistate cluster of Salmonella enterica serotype Oslo infections with an indistinguishable pulse-field gel electrophoresis pattern (Xbal PFGE pattern OSLX01.0090) was detected, involving 14 patients in eight states with illness onsets occurring during March 21-April 9. Epidemiologic evidence suggested that Persian cucumbers were the source of the outbreak; however, Salmonella was not isolated from any cucumbers.

What are the implications for public health practice?

Cucumbers have been identified as the source of several recent multistate outbreaks of Salmonella infections. As a consequence of these outbreaks, the Food and Drug Administration has selected cucumbers for an enhanced microbiologic surveillance sampling program for FY2016, in which both imported and domestic cucumbers will be tested for Salmonella and other pathogens. This program will assess whether any common factors are associated with Salmonella contamination. Implementation of new Food Safety Modernization Act requirements intended to prevent and minimize contamination of produce with pathogens might help to prevent or minimize future cucumber-associated Salmonella outbreaks.

characterize the genetic relatedness of bacteria isolated from patients. High quality single nucleotide polymorphism (hqSNP) analysis revealed all four clinical isolates from cases in Michigan and Ohio were highly related (differing by $0-1$ SNPs).

\section{Discussion}

Salmonella is the most common bacterial cause of foodborne disease in the United States and results in the highest number of hospitalizations and deaths among foodborne pathogens (1). Epidemiologic data indicate that Persian cucumbers were the source of Salmonella Oslo infections in this outbreak. Cucumbers were the only food eaten by patients significantly more often than expected. Further, most ill persons purchased a specific variety of cucumbers (Persian) from a single grocery chain. However, investigation into the source of these cucumbers did not find a common grower or other potential point of contamination.

This report highlights some of the inherent difficulties associated with outbreak investigations in which relatively short shelf life produce items are suspected. Given that the typical shelf life of cucumbers is 10-14 days, suspected cucumbers 


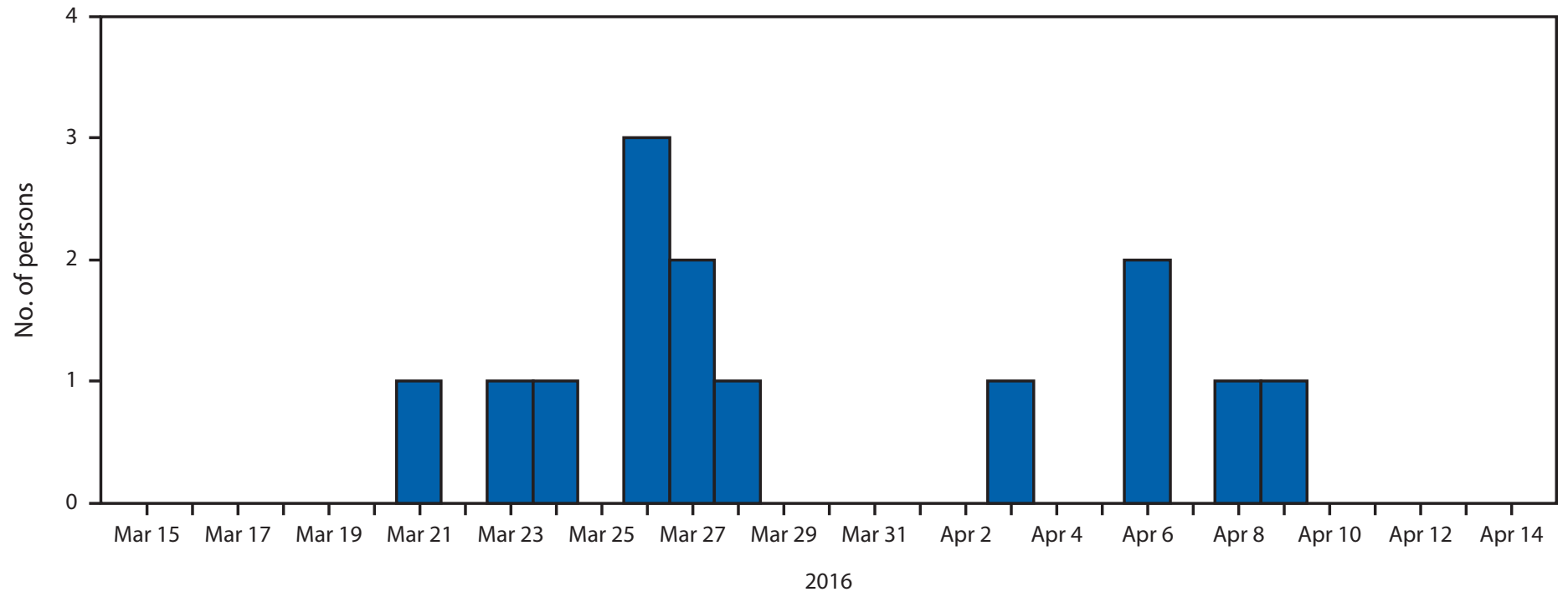

Date of illness onset

* Illinois, Kentucky, Massachusetts, Michigan, Minnesota, Nebraska, Ohio, and Wisconsin.

were no longer available in homes at the time ill persons were interviewed. In addition, Salmonella was not isolated from any cucumbers collected from ill persons or grocery stores, although the samples collected from points of sale and distribution might not have originated from the same farm as those consumed by persons before illness onset. However, despite being unable to test cucumbers earlier and find the outbreak strain of Salmonella, the epidemiologic evidence pointing to Persian cucumbers as the source of the outbreak was strong.

This is the fourth Salmonella outbreak since 2013 associated with cucumbers, with over 1,200 illnesses and 260 hospitalizations included in the previous three outbreaks (2-4). Two of these outbreaks were caused by cucumbers sourced from Mexico (2,4), whereas the other outbreak identified cucumbers sourced from Maryland as a major cause of illnesses (3). This outbreak supports the continued evaluation and sampling of produce by FDA with the Food Safety Modernization Act requirements intended to prevent and minimize contamination of produce with pathogens. Because the prevalence of Salmonella in cucumbers is unknown, FDA has initiated an enhanced sampling program for both domestic and imported whole, fresh, raw cucumbers within fiscal year 2016. The data (approximately 380 domestic cucumber samples and more

\footnotetext{
http://www.fda.gov/Food/GuidanceRegulation/FSMA/ucm361902.htm.
}

than 1,200 imported cucumber samples) will suggest whether any common factors, such as season, region, and whether the product was produced domestically or imported, are associated with Salmonella contamination.

Recent outbreaks have used industry consultations to help provide clues to focus the investigation, so information about cucumber harvesting and distribution was readily available. Early identification and prompt investigation of this outbreak while it was still occurring was important because it enabled investigators to present evidence to chain $\mathrm{A}$, a national grocer. Chain A's swift action to remove all Persian cucumber products, in addition to the short shelf life of cucumbers, likely contributed to the small size and short duration of this outbreak. Quick action by industry is essential to control future outbreaks. Continued communication between state and federal agencies and implicated retail locations and industry can also enhance the timeliness of response to effectively end outbreaks.

Consumers and retailers should always follow safe produce handling recommendations.** Cucumbers, like most produce, should be washed thoroughly, scrubbed with a clean produce brush before peeling or cutting, and refrigerated as soon as possible to prevent multiplication of bacteria such as Salmonella.

\footnotetext{
** http://www.foodsafety.gov/keep/types/fruits/tipsfreshprodsafety.html.
} 


\section{Acknowledgments}

Tyann Blessington, PhD, Sheila Merriweather, MPH, Food and Drug Administration, Silver Spring, Maryland; Illinois Department of Public Health; Kentucky Department for Public Health; Nebraska Department of Health and Human Services; Ohio Department of Health; Wisconsin Department of Health Services; Food and Drug Administration Coordinated Outbreak Response and Evaluation Network, Silver Spring, Maryland; Division of Foodborne, Waterborne, and Environmental Diseases, National Center for Emerging and Zoonotic Infectious Diseases, CDC.

\footnotetext{
${ }^{1}$ Division of Foodborne, Waterborne, and Environmental Diseases, National Center for Emerging and Zoonotic Infectious Diseases, CDC; ${ }^{2}$ Minnesota Department of Health; ${ }^{3}$ Minnesota Department of Agriculture; ${ }^{4}$ Michigan Department of Health and Human Services; ${ }^{5}$ Massachusetts Department of Public Health; ${ }^{6}$ Coordinated Outbreak Response and Evaluation Network, Food and Drug Administration, Silver Spring, Maryland.
}

Corresponding author: Lyndsay Bottichio, xmm8@cdc.gov, 404-639-0570.

\section{References}

1. CDC. Burden of foodborne illness: findings. Atlanta, GA: US Department of Health and Human Services, CDC; 2016. http://www.cdc.gov/ foodborneburden/2011-foodborne-estimates.html

2. CDC. Multistate outbreak of Salmonella Saintpaul infections linked to imported cucumbers (final update). Atlanta, GA: US Department of Health and Human Services, CDC; 2013. http://www.cdc.gov/ salmonella/saintpaul-04-13/

3. Angelo KM, Chu A, Anand M, et al. Outbreak of Salmonella Newport infections linked to cucumbers-United States, 2014. MMWR Morb Mortal Wkly Rep 2015;64:144-7.

4. CDC. Multistate outbreak of Salmonella Poona infections linked to imported cucumbers (final update). Atlanta, GA: US Department of Health and Human Services, CDC; 2016. http://www.cdc.gov/ salmonella/poona-09-15/ 